\title{
Optimal backward light scattering by dipolar particles
}

\author{
Jorge Olmos-Trigo $\odot,{ }^{1, *}$ Diego R. Abujetas $\odot,{ }^{1,2}$ Cristina Sanz-Fernández $\odot,{ }^{3}$ José A. Sánchez-Gil $\odot,{ }^{2}$ and Juan José Sáenz $\odot^{1,4}$ \\ ${ }^{1}$ Donostia International Physics Center (DIPC), 20018 Donostia-San Sebastián, Spain \\ ${ }^{2}$ Instituto de Estructura de la Materia (IEM-CSIC), Serrano 121, 28006 Madrid, Spain \\ ${ }^{3}$ Centro de Física de Materiales (CFM-MPC), Centro Mixto CSIC-UPV/EHU, Consejo Superior de Investigaciones Científicas, \\ 20018 Donostia-San Sebastián, Spain \\ ${ }^{4}$ IKERBASQUE, Basque Foundation for Science, 48013 Bilbao, Spain
}

(Received 26 June 2019; accepted 28 January 2020; published 27 February 2020)

\begin{abstract}
The near-zero forward intensity condition for light scattering by a dielectric dipolar sphere is usually associated with the generalized second Kerker condition, at which equal amplitude electric and magnetic dipolar responses are phase-shifted by $\pi$. As we show, this condition does lead to optimal backward light scattering for a given scattering cross section. However, it is clearly insufficient to give rise to the nearly zero optical forward scattering, in striking contrast to the actual view of the problem. In fact, we show that the generalized second Kerker condition leads to an energy radiation pattern that ranges all possible optical scattering diagrams depending on the total scattering cross section. Interestingly, we demonstrate that optimization of backward intensity, near the electric and magnetic dipolar resonances, leads to the counterintuitive result of a far-field energy radiation pattern with nearly zero backscattering.
\end{abstract}

DOI: 10.1103/PhysRevResearch.2.013225

The conditions of perfect zero light scattering from magnetic spheres were brought to the physical scene by Kerker, Wang and Giles [1] by assuming magnetodielectric spheres with a particular combination of relative electric permittivity $\epsilon$ and magnetic permeability $\mu$. They proved that when $\epsilon=\mu$, the backscattered radiation from the sphere is identical to zero. In contrast, for very small particles, when $\epsilon=(4-\mu) /(2 \mu+1)$, the forward scattering was shown to be reduced to zero, except for the peculiar case of $\epsilon=\mu=-2$ [2].

The study of these two optical responses, often referred to as Kerker conditions and, in particular, the inconsistency between the zero-forward condition and the optical theorem, have attracted a great interest [2-6]. The apparent paradox was solved by Alù and Engheta [5] by introducing the concept of near-zero-forward condition for magnetic Rayleigh particles. Kerker conditions, originally discussed for hypothetical magnetodielectric particles, were later shown to apply to subwavelength dielectric $(\mu=1)$ particles of high refractive index (HRI) materials [7,8]. Remarkably, the scattering properties of these HRI nano-particles are given by their dipolar electric and magnetic responses [9-14].

For HRI subwavelength isotropic spheres, the scattering can be fully described by the first electric, $a_{1}$, and magnetic, $b_{1}$, Mie coefficients [15],

$$
a_{1}=i \sin \alpha_{1} e^{-i \alpha_{1}}, \quad b_{1}=i \sin \beta_{1} e^{-i \beta_{1}},
$$

\footnotetext{
*jolmostrigo@gmail.com

Published by the American Physical Society under the terms of the Creative Commons Attribution 4.0 International license. Further distribution of this work must maintain attribution to the author(s) and the published article's title, journal citation, and DOI.
}

where the phase-shifts $\alpha_{1}$ and $\beta_{1}$ are real in the absence of absorption. Under plane wave illumination, the intensity in the backscattering direction can be exactly zero whenever $a_{1}=$ $b_{1} \Leftrightarrow \alpha_{1}=\beta_{1}$, independently of any other particle's material property [7]. At this condition, the particle's optical response consists of equal amplitude crossed electric- and magneticinduced dipoles oscillating in-phase (first Kerker condition), leading to destructive interference between scattered fields in backscattering. This prediction was experimentally demonstrated first for millimeter-scale ceramic spheres in the microwave regime [16] and shortly after for nanometer-scale HRI Si [17] and GaAs [18] nanospheres. Since then, the study of Kerker conditions has been significantly expanded and generalized, becoming ubiquitous in nano-optics and photonics [19]. New Kerker phenomena have been shown to emerge from the interplay between dipolar and higher order multipolar responses [20-22], from nonspherical particle's shapes [23-25] or from near-field [26,27] and structured beam illumination [28-31]. The relationship between electromagnetic duality symmetry and Kerker conditions [32,33], the role of the optical helicity [34] and light spin-orbit interactions near the first Kerker condition [35] are being topics of intense research activity. Interestingly, these phenomena are as well related to the emergence of spin-orbit optical mirages [36,37] and spin-momentum locking phenomena [38].

In contrast with the first Kerker condition, the zero-forward condition, which arises when the second Kerker condition is satisfied in the dipolar regime, $a_{1}=-b_{1}$, is strictly inhibited by the optical theorem [5]. As an alternative definition, we can consider the generalized second Kerker condition (GSKC) [7], $\alpha_{1}=-\beta_{1}$, corresponding to crossed electric and magnetic dipoles of equal amplitude oscillating anti-phase. A strong suppression of forward scattering was already experimentally observed in the microwave regime [16] at this condition, in 
agreement with the near-zero-forward intensity condition for Rayleigh particles [5,7]. A strong suppression of forward scattering is an important issue in light transport and scattering in nanostructured complex media [39-48], being also relevant in the discussion of the, so-called, anapole modes [19,49,50], as well as in the context of optical forces [51-55]. In most of the above-mentioned works, the equivalence between the GSKC and the near zero-forward condition was taken from granted even though it was only demonstrated in the Rayleigh limit, where the particle scattering cross sections are very small.

In this paper, we demonstrate that the GSKC (and the first Kerker condition) for dipolar particles can be formally derived as the optimal combination of electric and magnetic dipolar responses leading to maximum backward (and forward) scattered intensities for a fixed scattering cross section in the absence of optical gain. As expected, the maximum forward intensity takes place at $\alpha_{1}=\beta_{1}$ and correspond to the zero-backward condition, independently of the scattering amplitudes. The optimal backward scattering takes place at $\alpha_{1}=-\beta_{1}$ (i.e., the GSKC) but, interestingly, it does not lead to the near-zero optical forward condition for strong scattering regimes. Our results reveal that, in the dipolar regime, the GSKC straightforwardly returns the minimum asymmetry parameter ( $g$ parameter) for a fixed scattering cross section in the absence of optical gain. However, the $g$ parameter, defined as the average of the cosine of the scattering angle $g=\langle\cos \theta\rangle$ [39], is not always negative at the GSKC. As a result, the differential scattering cross section or, in other words, the redistribution of energy in the far-field limit, ranges all possible scattering angle diagrams. In particular, our results provide a consistent explanation of the intriguing exception for zero-forward scattering predicted for small, $\varepsilon=\mu=-2$, magnetic particles [2]: when the scattering cross section approaches its maximum value (when the electric and magnetic dipolar resonances are excited simultaneously), the differential scattering cross section resembles the one given by the first Kerker condition, in opposition to the physical insight given thus far.

The electromagnetic fields scattered by HRI dielectric nanoparticles present curious properties arising from the interference between the electric and magnetic multipoles. Most of them are embedded in the $g$ parameter, calculated from the differential scattering cross section, as [15]

$$
g=\langle\cos \theta\rangle=\frac{\int \frac{d \sigma_{\mathrm{s}}}{d \Omega} \cos \theta d \Omega}{\int \frac{d \sigma_{\mathrm{s}}}{d \Omega} d \Omega}=\frac{\int \frac{d \sigma_{\mathrm{s}}}{d \Omega} \cos \theta d \Omega}{\sigma_{\mathrm{s}}},
$$

where $\sigma_{\mathrm{s}}$ is the scattering cross section. For dipolar scatterers it is easy to show that the differential scattering cross section is given by [36]

$$
\frac{d \sigma_{\mathrm{s}}(\theta)}{d \Omega}=\frac{3}{8 \pi} \sigma_{\mathrm{s}}\left(\frac{1+\cos ^{2} \theta}{2}+2 g \cos \theta\right)
$$

where

$$
g=\frac{\Re\left\{a_{1} b_{1}^{*}\right\}}{\left|a_{1}\right|^{2}+\left|b_{1}\right|^{2}}=\frac{\sin \alpha_{1} \sin \beta_{1} \cos \left(\alpha_{1}-\beta_{1}\right)}{\sin ^{2} \alpha_{1}+\sin ^{2} \beta_{1}},
$$

and

$$
\sigma_{\mathrm{s}}=\frac{6 \pi}{k^{2}}\left(\left|a_{1}\right|^{2}+\left|b_{1}\right|^{2}\right)=\frac{6 \pi}{k^{2}}\left(\sin ^{2} \alpha_{1}+\sin ^{2} \beta_{1}\right) .
$$

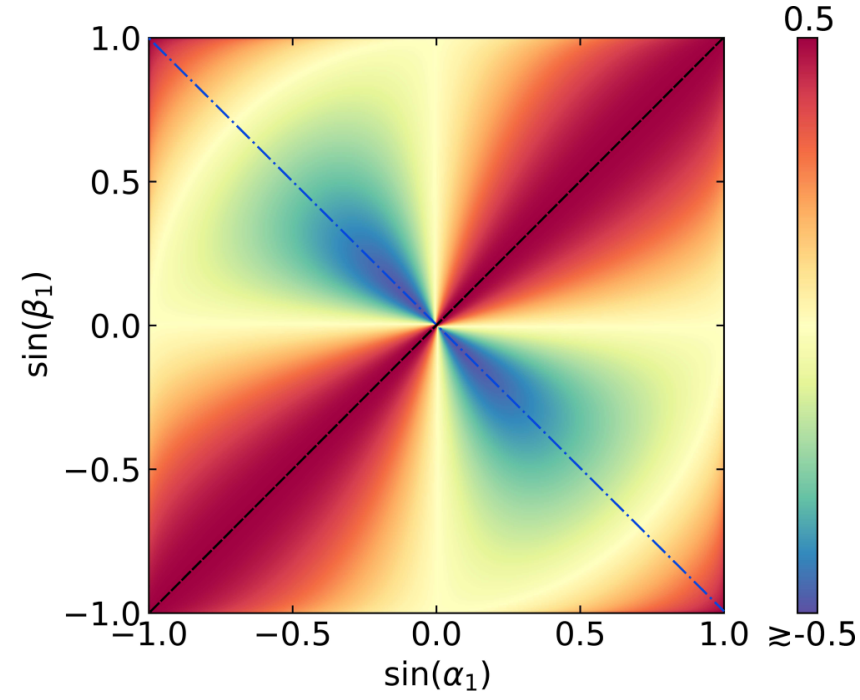

FIG. 1. Asymmetry parameter $g$ as a function of the dipolar electric and magnetic scattering phase shifts, $\alpha_{1}$ and $\beta_{1}$, respectively. The first Kerker condition (black dashed line), given by $\alpha_{1}=\beta_{1}$, gives rise to the maximum value of the $g$ parameter, regardless of the total scattering cross section. As seen in the attached scale, the GSKC (blue dash-dotted line), given by $\alpha_{1}=-\beta_{1}$, minimizes the $g$ parameter for a fixed scattering cross section, $\sigma_{\mathrm{s}}$ [see Eq. (5)]. The minimum value, $g=-1 / 2$, cannot be reached due to causality.

From Eqs. (3) and (4), it is trivial to see that, at the first Kerker condition, i.e., $g=1 / 2 \Leftrightarrow \alpha_{1}=\beta_{1}$, the differential scattering cross section vanishes at backscattering, i.e., $d \sigma_{\mathrm{s}}(\pi) / d \Omega=0$, regardless of the total scattering cross section, $\sigma_{\mathrm{s}}$. Interestingly, it can be shown that this does not depend on the incident polarization for spheres [56]. On the other hand, the optical response given by $a_{1}=-b_{1}$ would lead to the minimum analytical value of the $g$ parameter in the dipolar regime, $g=-0.5$ [57-59]. This in turn, would give rise to the zero forward scattering condition, $d \sigma_{\mathrm{s}}(0) / d \Omega=0$, according to Eq. (3). However, this condition is prohibited due to the optical theorem [5], which guarantees that the real part of the Mie coefficients must be positive-definite [60]. On these bases, a new energetically viable GSKC was proposed by Nieto-Vesperinas et al.: $\alpha_{1}=-\beta_{1}$ [7]. So far, this was thought to be the optimized condition that gives rise to a negative $g$ parameter, which in turn might reduce the scattered light in the forward direction [61]. Surprisingly, it is straightforward to notice via Eq. (4) that the GSKC does not (generally) lead to a negative $g$ parameter,

$$
\alpha_{1}=-\beta_{1} \quad \Rightarrow \quad g=\frac{1}{2}\left[\frac{k^{2}}{6 \pi} \sigma_{\mathrm{s}}^{K_{2}}-1\right],
$$

where $\sigma_{\mathrm{s}}^{K_{2}}=\left(12 \pi / k^{2}\right) \sin ^{2} \alpha_{1}$, according to Eq. (5).

This is the first important result of the present work. As it can be seen from Eqs. (5) and (6), only a relatively weak scattering leads to negative values of the $g$ parameter. The threshold, i.e., $g=0$, is given by $\pm \alpha_{1}=\mp \beta_{1}=\pi / 4$. Interestingly, this value corresponds to the scattering cross section that arises from a pure dipolar electric (or magnetic) resonant particle, $\sigma_{\mathrm{s}}^{\text {res }}=6 \pi / k^{2}$. 


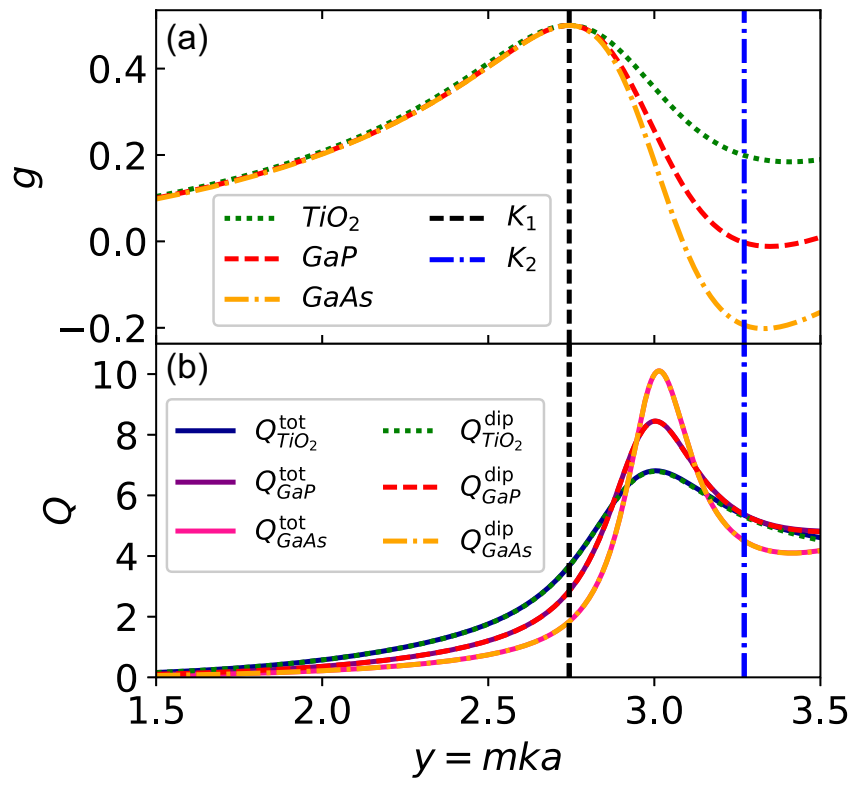

FIG. 2. (a) Asymmetry parameter $g$ as a function of the $y=m k a$ size parameter, where $m$ is the relative refractive index, $k$ is the wave vector in the medium and $a$ is the particle's radius. The black dashed and blue dash-dotted lines specifies the first Kerker and the GSKCs, respectively. At the generalized second Kerker condition $\left(K_{2}\right)$, the $g$ parameter is negative for the Gallium Arsenide-like sphere (GaAs), roughly zero for the Gallium phosphidelike sphere $(\mathrm{GaP})$ case, while it is positive for the Titanium Oxide-like sphere $\left(\mathrm{TiO}_{2}\right)$. (b) Scattering efficiencies arising from both a dipolar and a fully multipolar optical response, $Q^{\text {tot }}$ and $Q^{\text {dip }}$, respectively. As can be seen, the optical response is purely dipolar for the $\mathrm{GaAs}, \mathrm{GaP}$ and $\mathrm{TiO}_{2}$ spheres in this spectral range.

This phenomenology can be inferred from Fig. 1, where the $g$ parameter is illustrated as a function of the dipolar electric and magnetic scattering phase-shifts. Notice that Fig. 1 covers all possible optical responses in the dipolar regime as they run over all possible values of the first electric and magnetic Mie coefficients, according to Eq. (1). It is important to recall that the Mie coefficients can be generalized for any geometry [62]. As predicted, the first Kerker condition $\left(\alpha_{1}=\beta_{1}\right)$ gives rise to the maximum value of the $g$ parameter, $g=0.5$. In addition, this is completely independent of the scattering cross section, $\sigma_{\mathrm{s}}$, which would correspond to circles in the figure, according to Eq. (5). Interestingly, it can be inferred that the GSKC, $\alpha_{1}=-\beta_{1}$, minimizes the $g$ parameter for a fixed scattering cross section. However, this is not sufficient to state that this condition always leads to negative values of the $g$ parameter.

As an illustrative example that confirms the previous statement, the $g$ parameter is considered for three spheres of different materials, titanium oxide-like $\left(\mathrm{TiO}_{2}, m=2.6\right)$, gallium phosphide-like (GaP, $m=3.1$ ), and gallium arsenidelike (GaAs, $m=3.6$ ) in air, as it can be seen from Fig. 2(a). Refractive index data for these materials were taken from [63]. This figure shows that, at the first Kerker condition, $\alpha_{1}=$ $\beta_{1}$, the maximum value of the asymmetry is always reached (dashed vertical black line). On the other hand, at the GSKC, $\alpha_{1}=-\beta_{1}$, which corresponds to the dash-dotted vertical blue line, the $g$ parameter is not always negative. In fact, the $g$ parameter is positive for the $\mathrm{TiO}_{2}$-like sphere, almost zero for the GaP-like sphere case while it becomes negative for the GaAs-like sphere. According to Eq. (6), this change of sign depends strongly on the strength of the scattering cross section. The scattering efficiency, $Q=\sigma_{\mathrm{s}} / \pi a^{2}$, is depicted in Fig. 2(b) assuming both a dipolar response $(l=1)$ and the full Mie multipolar expansion. As can be inferred, the optical responses are purely dipolar, even for strong scattering regimes.

Let us now analyze the relevance of the GSKC in the nearly-zero optical forward scattering condition, where

$$
\frac{d \sigma_{\mathrm{s}}(\theta)}{d \Omega}=\frac{3}{8 \pi} \sigma_{\mathrm{s}}^{K_{2}}\left[\frac{1+\cos ^{2} \theta}{2}+\left(\frac{k^{2}}{6 \pi} \sigma_{\mathrm{s}}^{K_{2}}-1\right) \cos \theta\right] .
$$

At the GSKC, $\alpha_{1}=-\beta_{1}$, the scattering cross section, $\sigma_{\mathrm{s}}^{K_{2}}$, governs the far-field pattern of the differential scattering cross section. As a consequence, the GSKC is not sufficient (not even necessary) to obtain the typical pear-like structure, in striking contrast to previous analysis [8,64]. In fact, it is clear that near the electric and magnetic dipole resonances, in which $g \lesssim 0.5 \Leftrightarrow \sigma_{\mathrm{s}}^{K_{2}} \lesssim 12 \pi / k^{2}$, the radiation pattern of the differential scattering cross section resembles the one arising when the first Kerker condition is satisfied. In this particular case, there is no net radiation at backscattering. On the other hand, when $\sigma_{\mathrm{s}}^{K_{2}}=\sigma_{\mathrm{s}}^{\text {res }}=6 \pi / k^{2}$, condition that leads to $g=$ 0 , according to Eq. (6), the differential scattering cross section is identical to the one given by a pure electric (or magnetic) dipole. Finally, when $\sigma_{\mathrm{s}}^{K_{2}}<\sigma_{\mathrm{s}}^{\text {res }}$, which implies a negative $g$ parameter, the target scatters mostly in the backward direction.

This phenomenology is further confirmed in Fig. 3, where the far field radiation pattern of the (integral normalized) differential scattering cross section is considered, at the GSKC, for the materials illustrated in Fig. 2: $\mathrm{TiO}_{2}$-like, GaP-like, and GaAs-like spheres. As expected, when $g>0$, the scattering pattern almost entirely lies in the forward direction, according to Fig. 3(a). This corresponds to the energy radiation pattern in the far field limit of the $\mathrm{TiO}_{2}$-like sphere at the GSKC, where $g=0.2$, (see dotted green line Fig. 2). At $g=0$, which arises from the GaP-like sphere at the GSKC, according to the dashed red line in Fig. 2, the energy radiation pattern in the far field limit is symmetrical, as can be seen in Fig. 3(b). This is identical to the one that arises from a pure electric (or magnetic) dipole. Finally, when $g<0$, the target preferentially scatters in the backward direction, as can be inferred in Fig. 3(c). This phenomenon corresponds to the energy radiation pattern in the far field limit of the GaAs-like sphere at the GSKC, where $g=-0.2$, according to the dash-dotted yellow line of Fig. 2. From our analysis it is straightforward to derive that the nearly zero optical forward scattering condition is only achievable through a negative $g$ parameter.

In order to get deeper physical insight into the relevance of the GSKC, it is interesting to derive the explicit expression of the differential scattering cross section evaluated at the forward direction, as shown in Fig. 3. Under these conditions,

$$
\begin{gathered}
\alpha_{1}=-\beta_{1}, \\
\theta=0,
\end{gathered} \Rightarrow \frac{d \sigma_{\mathrm{s}}}{d \Omega}=\frac{k^{2}}{16 \pi}\left(\sigma_{\mathrm{s}}^{K_{2}}\right)^{2} .
$$




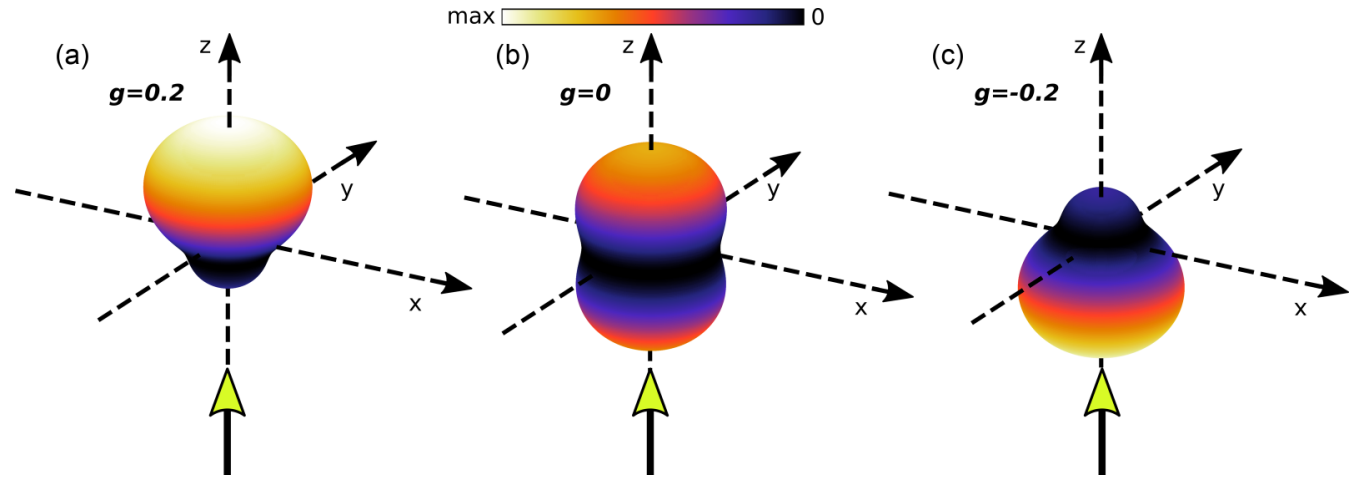

FIG. 3. (Integral-normalized) differential scattering cross section, $\left(d \sigma_{\mathrm{s}}(\theta) / d \Omega\right) / \sigma_{\mathrm{s}}^{K_{2}}$, for different optical responses at the generalized second Kerker conditon, i.e., $g=0.2$ ( $\mathrm{TiO}_{2}$-like sphere), $g=0$ (GaP-like sphere), $g=-0.2$ (GaAs-like sphere), according to Fig. 2. The incident light is illustrated by a vertical yellow arrow.

Equation (8) shows that at the GSKC, in the forward direction $(\theta=0)$, the differential scattering cross section scales quadratically with the scattering cross section. As a direct result, for a relatively large scattering cross section, the target may scatter preferentially in the forward direction $(\theta=0)$, leading to a counterintuitive result as the nearly-zero optical forward intensity condition for light is far from being obtained. In fact, near the electric and magnetic dipolar resonances, where $\pm \alpha_{1}, \mp \beta_{1} \approx \pi / 2 \Leftrightarrow g \lesssim 0.5$, the intensity in the forward direction $(\theta=0)$ is close to be maximized. Therefore both Figs. 1 and 4 can be understood together as the actual implication of the GSKC: Only when the scattering cross section is smaller than that for a pure resonant particle, $\sigma_{\mathrm{s}}^{K_{2}}<\sigma_{\mathrm{s}}^{\text {res }}$, a negative $g$ parameter can be obtained. In this regime and only in this regime, the nearly-zero optical forward scattering can be achieved.

In conclusion, we have shown that the GSKC can be derived as the optimal condition that minimizes the asym-

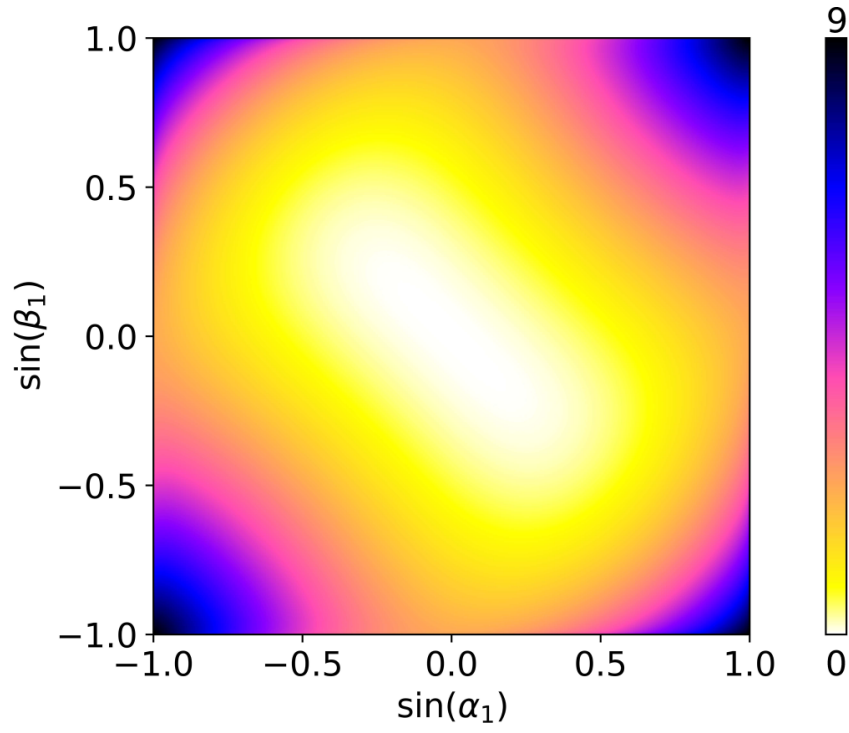

FIG. 4. Dimensionless differential scattering cross section, $k^{2} d \sigma_{\mathrm{s}} / d \Omega$, evaluated at the forward direction, $\theta=0$. At the GSKC, $\alpha_{1}=-\beta_{1}$, near the electric and magnetic resonances, the differential scattering cross section is maximized. When the scattering cross section is relatively small, it reaches its minimum value. metry, in terms of the $g$ parameter, for a fixed scattering cross section in the absence of optical gain. Interestingly, we have found that the GSKC does not necessarily give rise to a negative $g$ parameter. In fact, under this condition, we have demonstrated that the $g$ parameter ranges from positive to negative values, crossing $g=0$ when the scattering cross section is identical to the one arising from a pure electric (or magnetic) resonant target. As a direct consequence, we have demonstrated that the far-field scattering pattern of the differential scattering cross section runs over all its possible polar diagrams. Notably, near the electric and magnetic dipole resonances, we have explicitly exposed that this resembles the one given at the first Kerker condition, where there is no net radiation in the backscattering direction. This phenomenon implies the opposite physical insight which was assumed in previous works. In order to have a deeper insight, we have then confirmed this behavior by showing that the energy radiation pattern in the far-field limit, or, in other words, the differential scattering cross section, at the GSKC in the forward direction, scales quadratically with the scattering cross section. As a direct consequence, for strong scattering regimes, the target preferably scatters in the forward direction, in striking contrast with the current understanding.

These findings are of considerably importance in different contexts where minimization of the $g$ parameter can be most relevant. This includes theory and experimental work on optical forces (where the radiation pressure cross section strongly depends on the $g$ parameter), light transport and radiative transfer phenomena since the minimization of $g$ can significantly reduce the transport mean free path. We do then believe that our straightforward but fundamental analysis provides new insight in the study of light scattering from nanostructures which can be relevant in understanding more complex, multiple scattering processes in nanostructured samples and photonic devices.

This research was supported by the Basque Government (Project PI-2016-1-0041 and PhD Fellowship PRE-20182-0252) and by the Spanish MINECO and MICINN and European Regional Development Fund (ERDF) Projects: FIS2015-69295-C3-2-P, FIS2015-69295-C3-3-P, FIS201791413-EXP， FIS2017-82804-P， PGC2018-095777-B-C21, and PhD Fellowship FPU15/03566. 
[1] M. Kerker, D.-S. Wang, and C. Giles, Electromagnetic scattering by magnetic spheres, J. Opt. Soc. Am. A 73, 765 (1983).

[2] B. García-Cámara, F. González, F. Moreno, and J. M. Saiz, Exception for the zero-forward-scattering theory, J. Opt. Soc. Am. A 25, 2875 (2008).

[3] R. V. Mehta, R. Patel, R. Desai, R. V. Upadhyay, and K. Parekh, Experimental Evidence of Zero Forward Scattering by Magnetic Spheres, Phys. Rev. Lett. 96, 127402 (2006); B. García-Cámara, F. Moreno, F. González, and J. M. Saiz, Comment on "Experimental Evidence of Zero Forward Scattering by Magnetic Spheres", ibid. 98, 179701 (2007); H. Ramachandran and N. Kumar, Comment on "Experimental Evidence of Zero Forward Scattering by Magnetic Spheres", ibid. 100, 229703 (2008)

[4] J. M. Laskar, J. Philip, and B. Raj, Light scattering in a magnetically polarizable nanoparticle suspension, Phys. Rev. E 78, 031404 (2008).

[5] A. Alù and N. Engheta, How does zero forward-scattering in magnetodielectric nanoparticles comply with the optical theorem? J. Nanophoton. 4, 041590 (2010).

[6] B. García-Cámara, R. A. de La Osa, J. Saiz, F. González, and F. Moreno, Directionality in scattering by nanoparticles: Kerker's null-scattering conditions revisited, Opt. Lett. 36, 728 (2011).

[7] M. Nieto-Vesperinas, R. Gomez-Medina, and J. J. Saenz, Angle-suppressed scattering and optical forces on submicrometer dielectric particles, J. Opt. Soc. Am. A 28, 54 (2011).

[8] R. Gomez-Medina, B. Garcia-Camara, I. Suárez-Lacalle, F. González, F. Moreno, M. Nieto-Vesperinas, and J. J. Sáenz, Electric and magnetic dipolar response of germanium nanospheres: Interference effects, scattering anisotropy, and optical forces, J. Nanophoton. 5, 053512 (2011).

[9] A. B. Evlyukhin, C. Reinhardt, A. Seidel, B. S. Luk'yanchuk, and B. N. Chichkov, Optical response features of Sinanoparticle arrays, Phys. Rev. B 82, 045404 (2010).

[10] A. García-Etxarri, R. Gómez-Medina, L. S. Froufe-Pérez, C. López, L. Chantada, F. Scheffold, J. Aizpurua, M. NietoVesperinas, and J. J. Sáenz, Strong magnetic response of submicron silicon particles in the infrared, Opt. Express 19, 4815 (2011).

[11] A. I. Kuznetsov, A. E. Miroshnichenko, Y. H. Fu, J. Zhang, and B. Luk'yanchuk, Magnetic light, Sci. Rep. 2, 492 (2012).

[12] L. Shi, T. U. Tuzer, R. Fenollosa, and F. Meseguer, A new dielectric metamaterial building block with a strong magnetic response in the sub-1.5-micrometer region: Silicon colloid nanocavities, Adv. Mater. 24, 5934 (2012).

[13] R. Alaee, M. Albooyeh, A. Rahimzadegan, M. S. Mirmoosa, Y. S. Kivshar, and C. Rockstuhl, All-dielectric reciprocal bianisotropic nanoparticles, Phys. Rev. B 92, 245130 (2015).

[14] A. I. Kuznetsov, A. E. Miroshnichenko, M. L. Brongersma, Y. S. Kivshar, and B. Luk'yanchuk, Optically resonant dielectric nanostructures, Science 354, aag2472 (2016).

[15] H. C. Hulst and H. C. van de Hulst, Light Scattering by Small Particles (Courier Corporation, 1957).

[16] J.-M. Geffrin, B. García-Cámara, R. Gómez-Medina, P. Albella, L. S. Froufe-Pérez, C. Eyraud, A. Litman, R. Vaillon, F. González, M. Nieto-Vesperinas, J. J. Sáenz, and F. Moreno, Magnetic and electric coherence in forward- and back-scattered electromagnetic waves by a single dielectric subwavelength sphere, Nat. Commun. 3, 1171 (2012).
[17] Y. H. Fu, A. I. Kuznetsov, A. E. Miroshnichenko, Y. F. Yu, and B. Luk'yanchuk, Directional visible light scattering by silicon nanoparticles, Nat. Commun. 4, 1527 (2013).

[18] S. Person, M. Jain, Z. Lapin, J. J. Sáenz, G. Wicks, and L. Novotny, Demonstration of zero optical backscattering from single nanoparticles, Nano Lett. 13, 1806 (2013).

[19] W. Liu and Y. S. Kivshar, Generalized Kerker effects in nanophotonics and meta-optics, Opt. Express 26, 13085 (2018).

[20] W. Liu, A. E. Miroshnichenko, R. F. Oulton, D. N. Neshev, O. Hess, and Y. S. Kivshar, Scattering of core-shell nanowires with the interference of electric and magnetic resonances, Opt. Lett. 38, 2621 (2013).

[21] W. Liu, J. Zhang, B. Lei, H. Ma, W. Xie, and H. Hu, Ultradirectional forward scattering by individual core-shell nanoparticles, Opt. Express 22, 16178 (2014).

[22] H. K. Shamkhi, K. V. Baryshnikova, A. Sayanskiy, P. Kapitanova, P. D. Terekhov, P. Belov, A. Karabchevsky, A. B. Evlyukhin, Y. Kivshar, and A. S. Shalin, Transverse Scattering and Generalized Kerker Effects in All-Dielectric Mie-Resonant Metaoptics, Phys. Rev. Lett. 122, 193905 (2019).

[23] B. S. Luk'yanchuk, N. V. Voshchinnikov, R. PaniaguaDomínguez, and A. I. Kuznetsov, Optimum forward light scattering by spherical and spheroidal dielectric nanoparticles with high refractive index, ACS Photon. 2, 993 (2015).

[24] Z. Wang, N. An, F. Shen, H. Zhou, Y. Sun, Z. Jiang, Y. Han, Y. Li, and Z. Guo, Enhanced forward scattering of ellipsoidal dielectric nanoparticles, Nanoscale Res. Lett. 12, 58 (2017).

[25] T. Shibanuma, P. Albella, and S. A. Maier, Unidirectional light scattering with high efficiency at optical frequencies based on low-loss dielectric nanoantennas, Nanoscale 8, 14184 (2016).

[26] B. Rolly, B. Stout, and N. Bonod, Boosting the directivity of optical antennas with magnetic and electric dipolar resonant particles, Opt. Express 20, 20376 (2012).

[27] M. Dubois, L. Leroi, Z. Raolison, R. Abdeddaim, T. Antonakakis, J. De Rosny, A. Vignaud, P. Sabouroux, E. Georget, B. Larrat, G. Tayeb, N. Bonod, A. Amadon, F. Mauconduit, C. Poupon, D. LeBihan, and S. Enoch, Kerker Effect in Ultrahigh-Field Magnetic Resonance Imaging, Phys. Rev. X 8, 031083 (2018).

[28] M. Neugebauer, P. Woźniak, A. Bag, G. Leuchs, and P. Banzer, Polarization-controlled directional scattering for nanoscopic position sensing, Nat. Commun. 7, 11286 (2016).

[29] A. Bag, M. Neugebauer, P. Woźniak, G. Leuchs, and P. Banzer, Transverse Kerker Scattering for Angstrom Localization of Nanoparticles, Phys. Rev. Lett. 121, 193902 (2018).

[30] S. Nechayev, J. S. Eismann, M. Neugebauer, P. Woźniak, A. Bag, G. Leuchs, and P. Banzer, Huygens' dipole for polarization-controlled nanoscale light routing, Phys. Rev. A 99, 041801(R) (2019).

[31] C. S. Fernández, J. O. Trigo, and J. J. Saenz, Multiple Kerker conditions in arbitrary dielectric spheres, arXiv:1904.06687.

[32] X. Zambrana-Puyalto, I. Fernandez-Corbaton, M. Juan, X. Vidal, and G. Molina-Terriza, Duality symmetry and Kerker conditions, Opt. Lett. 38, 1857 (2013).

[33] X. Zambrana-Puyalto, X. Vidal, M. L. Juan, and G. MolinaTerriza, Dual and anti-dual modes in dielectric spheres, Opt. Express 21, 17520 (2013).

[34] I. Fernandez-Corbaton, X. Zambrana-Puyalto, N. Tischler, X. Vidal, M. L. Juan, and G. Molina-Terriza, Electromagnetic 
Duality Symmetry and Helicity Conservation for the Macroscopic Maxwell's Equations, Phys. Rev. Lett. 111, 060401 (2013).

[35] J. Olmos-Trigo, C. Sanz-Fernández, F. S. Bergeret, and J. J. Sáenz, Asymmetry and spin-orbit coupling of light scattered from subwavelength particles, Opt. Lett. 44, 1762 (2019).

[36] J. Olmos-Trigo, C. Sanz-Fernández, A. García-Etxarri, G. Molina-Terriza, F. S. Bergeret, and J. J. Sáenz, Enhanced spinorbit optical mirages from dual nanospheres, Phys. Rev. A 99, 013852 (2019).

[37] J. Olmos-Trigo, C. Sanz-Fernández, D. R. Abujetas, A. GarcíaEtxarri, G. Molina-Terriza, J. A. Sánchez-Gil, F. S. Bergeret, and J. J. Sáenz, Role of the absorption on the spin-orbit interactions of light with Si nano-particles, J. Appl. Phys. 126, 033104 (2019).

[38] M. F. Picardi, A. V. Zayats, and F. J. Rodríguez-Fortuño, Janus and Huygens Dipoles: Near-Field Directionality Beyond Spin-Momentum Locking, Phys. Rev. Lett. 120, 117402 (2018).

[39] R. Gómez-Medina, L. S. Froufe-Pérez, M. Yépez, F. Scheffold, M. Nieto-Vesperinas, and J. J. Sáenz, Negative scattering asymmetry parameter for dipolar particles: Unusual reduction of the transport mean free path and radiation pressure, Phys. Rev. A 85, 035802 (2012).

[40] M. Liu, C. Zhao, and B. Wang, Polarization management based on dipolar interferences and lattice couplings, Opt. Express 26, 7235 (2018).

[41] B. X. Wang and C. Y. Zhao, Achieving a strongly negative scattering asymmetry factor in random media composed of dual-dipolar particles, Phys. Rev. A 97, 023836 (2018).

[42] A. Pors, S. K. Andersen, and S. I. Bozhevolnyi, Unidirectional scattering by nanoparticles near substrates: generalized Kerker conditions, Opt. Express 23, 28808 (2015).

[43] M. K. Schmidt, J. Aizpurua, X. Zambrana-Puyalto, X. Vidal, G. Molina-Terriza, and J. J. Sáenz, Isotropically Polarized Speckle Patterns, Phys. Rev. Lett. 114, 113902 (2015).

[44] R. R. Naraghi, S. Sukhov, and A. Dogariu, Directional control of scattering by all-dielectric core-shell spheres, Opt. Lett. 40, 585 (2015)

[45] M. Decker and I. Staude, Resonant dielectric nanostructures: A low-loss platform for functional nanophotonics, J. Opt. 18, 103001 (2016).

[46] E. E. Gorodnichev, A. I. Kuzovlev, and D. B. Rogozkin, Anomalous depolarizing properties of a disordered ensemble of resonant Mie particles, JETP Lett. 104, 157 (2016).

[47] P. Genevet, F. Capasso, F. Aieta, M. Khorasaninejad, and R. Devlin, Recent advances in planar optics: From plasmonic to dielectric metasurfaces, Optica 4, 139 (2017).

[48] Y. Kivshar and A. Miroshnichenko, Meta-optics with Mie resonances, Opt. Photon. News 28, 24 (2017).

[49] B. Luk'yanchuk, R. Paniagua-Domínguez, A. I. Kuznetsov, A. E. Miroshnichenko, and Y. S. Kivshar, Hybrid anapole modes of high-index dielectric nanoparticles, Phys. Rev. A 95, 063820 (2017).
[50] B. Luk'yanchuk, R. Paniagua-Domínguez, A. I. Kuznetsov, A. E. Miroshnichenko, and Y. S. Kivshar, Suppression of scattering for small dielectric particles: Anapole mode and invisibility, Philos. Trans. R. Soc. A 375, 20160069 (2017).

[51] M. Nieto-Vesperinas, J. Sáenz, R. Gómez-Medina, and L. Chantada, Optical forces on small magnetodielectric particles, Opt. Express 18, 11428 (2010).

[52] J. M. Auñón and M. Nieto-Vesperinas, Optical forces from evanescent bessel beams, multiple reflections, and Kerker conditions in magnetodielectric spheres and cylinders, J. Opt. Soc. Am. A 31, 1984 (2014).

[53] M. Nieto-Vesperinas, Optical torque: Electromagnetic spin and orbital-angular-momentum conservation laws and their significance, Phys. Rev. A 92, 043843 (2015).

[54] D. Gao, W. Ding, M. Nieto-Vesperinas, X. Ding, M. Rahman, T. Zhang, C. Lim, and C.-W. Qiu, Optical manipulation from the microscale to the nanoscale: Fundamentals, advances and prospects, Light Sci. Application 6, e17039 (2017).

[55] N. O. Länk, P. Johansson, and M. Käll, Directional scattering and multipolar contributions to optical forces on silicon nanoparticles in focused laser beams, Opt. Express 26, 29074 (2018).

[56] I. Fernandez-Corbaton, X. Zambrana-Puyalto, and G. MolinaTerriza, Helicity and angular momentum: A symmetry-based framework for the study of light-matter interactions, Phys. Rev. A 86, 042103 (2012).

[57] T. J. Arruda, A. S. Martinez, and F. A. Pinheiro, Electromagnetic energy and negative asymmetry parameters in coated magneto-optical cylinders: Applications to tunable light transport in disordered systems, Phys. Rev. A 94, 033825 (2016).

[58] T. J. Arruda, F. A. Pinheiro, and A. S. Martinez, Electromagnetic energy stored in inhomogeneous scattering systems, J. Opt. Soc. Am. A 34, 1934 (2017).

[59] R. Ali, F. A. Pinheiro, F. S. S. Rosa, R. S. Dutra, and P. A. M. Neto, Optimizing optical tweezing with directional scattering in composite microspheres, Phys. Rev. A 98, 053804 (2018).

[60] M. Nieto-Vesperinas, Optical theorem for the conservation of electromagnetic helicity: Significance for molecular energy transfer and enantiomeric discrimination by circular dichroism, Phys. Rev. A 92, 023813 (2015).

[61] B. García-Cámara, J. F. Algorri, A. Cuadrado, V. Urruchi, J. M. Sánchez-Pena, R. Serna, and R. Vergaz, All-optical nanometric switch based on the directional scattering of semiconductor nanoparticles, J. Phys. Chem. C 119, 19558 (2015).

[62] D. R. Abujetas, J. A. Sanchez-Gil, and J. J. Sáenz, Generalized brewster effect in high-refractive-index nanorod-based metasurfaces, Opt. Express 26, 31523 (2018).

[63] D. E. Aspnes and A. A. Studna, Dielectric functions and optical parameters of $\mathrm{Si}$, Ge, GaP, GaAs, GaSb, InP, InAs, and InSb from 1.5 to $6.0 \mathrm{eV}$, Phys. Rev. B 27, 985 (1983).

[64] B. García-Cámara, R. Gómez-Medina, J. J. Sáenz, and B. Sepúlveda, Sensing with magnetic dipolar resonances in semiconductor nanospheres, Opt. Express 21, 23007 (2013). 